\title{
PSEUDO-FACTORIALS, ELLIPTIC FUNCTIONS, AND CONTINUED FRACTIONS
}

\author{
ROLAND BACHER AND PHILIPPE FLAJOLET
}

\begin{abstract}
This study presents miscellaneous properties of pseudo-factorials, which are numbers whose recurrence relation is a twisted form of that of usual factorials. These numbers are associated with special elliptic functions, most notably, a Dixonian and a Weierstraß function, which parametrize the Fermat cubic curve and are relative to a hexagonal lattice. A continued fraction expansion of the ordinary generating function of pseudo-factorials, first discovered empirically, is established here. This article also provides a characterization of the associated orthogonal polynomials, which appear to form a new family of "elliptic polynomials", as well as various other properties of pseudo-factorials, including a hexagonal lattice sum expression and elementary congruences.
\end{abstract}

\section{Pseudo-factorials}

Start from the innocuous looking recurrence,

$$
\alpha_{n+1}=(-1)^{n+1} \sum_{k=0}^{n}\left(\begin{array}{l}
n \\
k
\end{array}\right) \alpha_{k} \alpha_{n-k}, \quad \alpha_{0}=1,
$$

which determines a sequence of integers with initial values

$$
1,-1,-2,2,16,-40,-320,1040,12160,-52480,-742400 .
$$

These numbers will be called pseudo-factorials, since the omission of the sign alternation $(-1)^{n+1}$ in Equation (1) determines the sequence of factorials, $0 !, 1 !, 2 !, \ldots$ At the suggestion of one of us (Bacher, October 2004), they have been included as Sequence A098777 in the On-line Encyclopedia of Integer Sequences [26], which is brilliantly maintained by Sloane and a gang of dedicated volunteers. The purpose of this note is to show that these numbers, though not classical, have a host of interesting properties.

The exponential generating function,

$$
f(z):=\sum_{n \geq 0} \alpha_{n} \frac{z^{n}}{n !}=1-z-z^{2}+\frac{z^{3}}{3}+2 \frac{z^{4}}{3}-\frac{z^{5}}{3}-\cdots,
$$

is fundamental to our treatment. (The fact that the absolute values $\left|\alpha_{n}\right|$ are dominated by factorials implies that $f(z)$ is analytic at least in $|z|<1$.) We first elucidate the relation between $f(z)$ and elliptic functions of a kind introduced by Alfred Cardew Dixon in 1890 (vide [7), then show the reduction to the more common Weierstraß framework of $\wp$-functions: this forms the subject of Sections 2 and 3. A simple consequence of the elliptic connections, worked out in Section 4 . is an expression of pseudo-factorials as sums over a hexagonal lattice. Next, we

Date: January 12, 2009; revised May 31, 2009. To appear in The Ramanujan Journal. 
establish a continued fraction of a new type, relative to the ordinary generating function of pseudo-factorials,

$$
F(z):=\sum_{n \geq 0} \alpha_{n} z^{n}=1-z-2 z^{2}+2 z^{3}+16 z^{4}-40 z^{5}-\cdots,
$$

to be taken in the sense of formal power series since its radius of convergence is 0 . To wit:

$$
F(z) \equiv \sum_{n \geq 0} \alpha_{n} z^{n}=\frac{1}{1+z+\frac{3 \cdot 1^{2} \cdot z^{2}}{1-z+\frac{2^{2} \cdot z^{2}}{1+3 z+\frac{3 \cdot 3^{2} \cdot z^{2}}{1-3 z+\frac{4^{2} \cdot z^{2}}{\ddots}}}}}
$$

where the denominators are successively $+1,-1,+3,-3,+5,-5, \ldots$, and the numerators are $3 \cdot 1^{2}, 2^{2}, 3 \cdot 3^{2}, 4^{2}, 3 \cdot 5^{2}, 6^{2}, \ldots$ Such a repetitive pattern of order 2 in what is known as a Jacobi fraction is somewhat unusual. The fraction (4) was first discovered experimentally - proving it in Sections 57 is the central theme of the present study.

Finally, the convergents of the continued fraction (4) can be made explicit, via generating functions: this is conducive to what seems to be a new class of "elliptic polynomials" in the sense of Lomont-Brillhart [17; see Section 8. In Section 9, we then draw several consequences of the previous developments, in the form of Hankel determinant evaluations and elementary congruence properties of pseudo-factorials.

Why? As kindly suggested by a referee and by the editor of The Ramanujan Journal, we offer a few comments relating to the motivations behind studies, such as the present one.

One primary motivation is to gain a better understanding of a still mysterious class of continued fractions, the ones that have coefficients that are polynomial (or rational) functions in the depth $n$. This highly general but somewhat impenetrable class of special functions seems to have been first noticed by Pollaczek in [22]. It is piquant to note that Apery's continued fraction [30, which first led to an irrationality proof of Riemann's $\zeta(3)$, has cubic denominators and sextic denominators, a feature also shared by the Conrad continued fractions [5, 6] relative to the Dixonian functions sm, cm, themselves closely related to pseudo-factorials (Section 2). Our main continued fraction (Theorem 5 is Pollaczek in a mildly extended sense: it has polynomial coefficients, but modulated by an unsual periodicity of order 2 .

On another register, the relation of elliptic functions to continued fractions is an old subject, going back at least to Eisenstein, Stieljes, Rogers, and Ramanujan. Whereas the $q$-series and $\vartheta$-function aspects are not immediately relevant to our discussion, we may observe that the continued fractions relative to the Jacobian functions sn, cn, dn have arithmetic content: together with the Hankel determinant product evaluations that they imply, they have been used by Milne in his penetrating study [20] of representations of integers as sums of $4 n^{2}$ or $4 n(n+1)$ square or triangular numbers. In this spirit, we offer some new Hankel determinant evaluations in Section 9, but will leave to others the task of determining whether they are of some arithmetic interest. 
The class of orthogonal polynomials associated to any continued fraction, whose coefficients are rational-in-the-depth, is also of interest. Many instances of low degree have been categorized by Chihara in [3. "Elliptic polynomials", that is, orthogonal polynomials associated with series expansions of elliptic functions have a tradition that goes back at least to Carlitz [2] and they form the topic of the entire book by Lomont and Brillhart [17]. The discovery of a new class (Section 8) is of interest in this context.

Finally, the pseudo-factorials are tightly coupled with $\frac{1}{\pi} \Gamma\left(\frac{1}{3}\right)^{3}$, as seen from our discussion of lattice sums in Section 4 . In this context, it is well worth noting a spectacular arithmetic continued fraction for $\Gamma\left(\frac{1}{3}\right)^{3}$, recently obtained by Tanguy Rivoal [23, which is of the sextic-duodecimic type(!). This and previous observations reflect the fact that our understanding of an orbit of questions, surrounding Pollaczek continued fractions, elliptic functions, elliptic polynomials, and diophantine approximation properties, is still fragmentary; but they also suggest that "hidden" structures are yet to be discovered in this area (see also our brief conclusion in Section 10p.

\section{Elliptic connections: Dixonian functions}

This section serves to establish the first connection between pseudo-factorials and elliptic functions. The starting point is the exponential generating function defined by (3); it satisfies a functional equation,

$$
f^{\prime}(z)=-f(-z)^{2},
$$

which directly translates the defining recurrence (1).

To make $f(z)$ explicit, take the functional equation (5) and differentiate once, so that

$$
f^{\prime \prime}(z)=2 f(-z) f^{\prime}(-z), \quad \text { implying } \quad f^{\prime \prime}(z)=-2 \sqrt{-f^{\prime}(z)} f(z)^{2},
$$

since, by (5) again, one has $f^{\prime}(-z)=-f(z)^{2}$ and $f(-z)=\sqrt{-f^{\prime}(z)}$. In order to solve the nonlinear differential equation, "cleverly" multiply by $\sqrt{-f^{\prime}(z)}$ to get

$$
f^{\prime \prime}(z) \sqrt{-f^{\prime}(z)}=2 f(z)^{2} f^{\prime}(z),
$$

which is integrated to give

$$
-\frac{2}{3}\left(-f^{\prime}(z)\right)^{3 / 2}=\frac{2}{3} f(z)^{3}-\frac{2}{3} K,
$$

with $K$ a yet unspecified constant. Equivalently, one has

$$
\frac{-f^{\prime}(z)}{\left(K-f(z)^{3}\right)^{2 / 3}}=1,
$$

which upon one more integration gives

$$
\int_{f(z)}^{1} \frac{d w}{\left(K-w^{3}\right)^{2 / 3}}=z,
$$

where use has been made of the initial condition $f(0)=1$. The constant $K$ is finally identified by means of a second order expansion (with $f^{\prime}(0)=-1, f^{\prime \prime}(0)=-2$ ), to the effect that one must have $K=2$. (The computations parallel those of [6].) 
In view of our subsequent treatment, it is convenient to standardize (9). A linear change of variables yields

$$
\int_{2^{-1 / 3} f(z)}^{2^{-1 / 3}} \frac{d y}{\left(1-y^{3}\right)^{2 / 3}}=2^{1 / 3} z
$$

where we have taken into account that $K=2$. Throughout this study, a fundamental constant is $\pi_{3}$ (a period of the function sm defined below in (14)),

$$
\pi_{3}:=3 \int_{0}^{1} \frac{d y}{\left(1-y^{3}\right)^{2 / 3}}=B\left(\frac{1}{3}, \frac{1}{3}\right)=\frac{\Gamma\left(\frac{1}{3}\right)^{2}}{\Gamma\left(\frac{2}{3}\right)}=\frac{\sqrt{3}}{2 \pi} \Gamma\left(\frac{1}{3}\right)^{3},
$$

where the evaluation results from the classical Eulerian integral [32, §12.4]:

$$
\mathrm{B}(\alpha, \beta):=\int_{0}^{1} t^{\alpha-1}(1-t)^{\beta-1} d t=\int_{0}^{\infty} \frac{u^{\alpha-1}}{(1+u)^{\alpha+\beta+1}} d u=\frac{\Gamma(\alpha) \Gamma(\beta)}{\Gamma(\alpha+\beta)} .
$$

(Numerically ${ }^{1}$, we find $\pi_{3} \doteq 5.2999162508$.) A simple computation shows that

$$
\int_{0}^{2^{-1 / 3}} \frac{d y}{\left(1-y^{3}\right)^{2 / 3}}=\frac{1}{6} B\left(\frac{1}{3}, \frac{1}{3}\right)=\frac{\pi_{3}}{6},
$$

so that we can write

$$
\int_{0}^{2^{-1 / 3} f(z)} \frac{d y}{\left(1-y^{3}\right)^{2 / 3}}=\frac{\pi_{3}}{6}-2^{1 / 3} z .
$$

The function $f(z)$ can now be expressed in terms of specific elliptic functions introduced by A. C. Dixon in his original memoir [7]. Define the function $\operatorname{sm}(z)$ by the equation

$$
\int_{0}^{\operatorname{sm}(z)} \frac{d y}{\left(1-y^{3}\right)^{2 / 3}}=z .
$$

Then, a comparison of $(13)$ and 14 permits us to identify $f(z)$.

Theorem 1. The exponential generating function of pseudo-factorials satisfies

$$
f(z)=2^{1 / 3} \operatorname{sm}\left(\frac{\pi_{3}}{6}-2^{1 / 3} z\right)
$$

where the Dixonian elliptic function $\operatorname{sm}(z)$ is as in (14) and $\pi_{3}$ is the constant (11).

We can offer a few comments regarding Dixonian functions. There is actually a pair of "higher-order trigonometric" functions, sm and $\mathrm{cm}$, where sm and $\mathrm{cm}$ are evocative of a sine and a cosine function, respectively. Their properties can be developed from first principles, as done by Dixon followed by Conrad-Flajolet [5. 6. 7], starting with the differential system,

$$
\operatorname{sm}^{\prime}(z)=\operatorname{cm}(z)^{2}, \quad \operatorname{cm}^{\prime}(z)=-\operatorname{sm}(z)^{2}
$$

and initial conditions $\operatorname{sm}(0)=0, \operatorname{cm}(0)=1$. (See also the works of Lundberg [18] and the recent developments by Lindquist and Peetre [15, 16, for a yet more general approach.) For the record, we note that

$\operatorname{sm}(z)=z-4 \frac{z^{4}}{4 !}+160 \frac{z^{7}}{7 !}-20800 \frac{z^{10}}{10 !}-\cdots, \quad \operatorname{cm}(z)=1-2 \frac{z^{3}}{3 !}+40 \frac{z^{6}}{6 !}-3680 \frac{z^{9}}{9 !}+\cdots$,

${ }^{1}$ The notation $x \doteq \xi$, with $\xi$ a decimal fraction, indicates that $\xi$ is an approximation of $x$ to the last digit stated. 
whose coefficients $(1,-4,160, \ldots$ and $1,-2,40, \ldots)$ are respectively $\mathbf{A} 104133$ and A104134 of Sloane's Encyclopedia. The works of Conrad and Flajolet [5, 6] provide continued fraction expansions for the ordinary generating function of these coefficients, but these are then relative to the expansions of sm, cm at 0 , and not at $\pi_{3} / 6$, as in $(15)$. Finally, we observe that, by the calculation $(7)$ and by (16), we have the identity

$$
\operatorname{sm}(z)^{3}+\operatorname{cm}(z)^{3}=1
$$

so that the pair $(\operatorname{sm}(z), \operatorname{cm}(z))$ parametrizes the Fermat cubic $\mathbf{F}_{\mathbf{3}}$ defined by the equation $X^{3}+Y^{3}=1$, which is of genus 1 .

\section{Elliptic connections: Weierstraß forms}

It is a priori possible to reduce the exponential generating function $f(z)$ of pseudo-factorials to any of the several canonical forms of elliptic functions. Here, we show, by elementary calculations similar to the ones of the previous section, how to arrive directly at an expression involving the Weierstraß function $\wp$. We recall that this function $\wp(z) \equiv \wp\left(z ; g_{2}, g_{3}\right)$ is classically defined by the nonlinear differential equation 32

$$
\wp^{\prime}(z)^{2}=4 \wp(z)^{3}-g_{2} \wp(z)-g_{3},
$$

together with the initial condition $\wp(z) \sim z^{-2}$ as $z \rightarrow 0$. By design, the pair $\left(\wp(z), \wp^{\prime}(z)\right)$ parametrizes the elliptic curve $Y^{2}=4 X^{3}-g_{2} X-g_{3}$, with invariants $g_{2}, g_{3}$.

The starting point is the fundamental relation (5), namely, $f^{\prime}(z)=-f(-z)^{2}$. We first claim the identity

$$
f(z)^{3}+f(-z)^{3}=2 .
$$

The proof is obtained by verifying that the derivative of the left-hand side is 0 ,

$$
\left(f(z)^{3}+f(-z)^{3}\right)^{\prime}=3 f(z)^{2} f^{\prime}(z)-3 f(-z)^{2} f^{\prime}(-z)=0,
$$

(the final reduction uses (5)), combined with the initial condition $f(0)=1$. Next, we have

$$
(-f(z) f(-z))^{\prime}=f(-z)^{3}-f(z)^{3},
$$

again by way of (5).

Now set

$$
g(z):=-f(z) f(-z)=-1+3 z^{2}-3 z^{4}+3 z^{6}-\frac{18}{7} z^{8}+\frac{15}{7} z^{10}-\frac{12}{7} z^{12}+\cdots .
$$

The basic elliptic connection is provided by the differential relation

$$
g^{\prime}(z)^{2}=4 g(z)^{3}+4,
$$

which is clearly of the Weierstraß type (17). To see it, it suffices to square the two sides of the identities (18) and (19), then compare the outcomes. Equation (20) then shows that $g(z)$ is closely related to the elliptic curve $\mathcal{E}$ defined by

$$
Y^{2}=4 X^{3}+4 \text {. }
$$


The curve $\mathcal{E}$ contains six integral points ${ }^{2}$ (including the point at infinity corresponding to the identity element of the underlying group) forming a cyclic group of order six. The non-trivial points of this group are: $(-1,0)$ (of order 2$),(0, \pm 2)$ (of order 3 ) and $(2, \pm 6)$ (of order 6 ). Since $\left(g(0), g^{\prime}(0)\right)=(-1,0)$, the series $g(z)$ represents the expansion of the Weierstraß function $\wp$ around the unique real 2-torsion point $(-1,0)$ of $\mathcal{E}$.

The following result recovers $f(z)$ from $g(z) \equiv-f(z) f(-z)$ and constitutes the main result of this section.

Theorem 2. Let $\wp(z):=\wp(z ; 0,-4)$ be the Weierstraß function with invariants $g_{2}=0$ and $g_{3}=-4$ and smallest positive real period ${ }^{3}$

$$
6 r=\pi_{3} 2^{-1 / 3}=\frac{2^{-4 / 3} 3^{1 / 2}}{\pi} \Gamma\left(\frac{1}{3}\right)^{3},
$$

with $\pi_{3}$ as in (11). The exponential generating function of pseudo-factorials satisfies

$$
f(i \sqrt{3} z)=\frac{-\wp^{\prime}(z+3 r)-2 i \sqrt{3}}{2 i \sqrt{3} \wp(z+3 r)} .
$$

Proof. We first establish the expression (22) of the real period of $\wp(z)$, only making use of the most basic properties of elliptic functions [32, Ch. XX]. Let us denote temporarily the real half-period by $\varpi$. By general properties of elliptic functions 4 we have $\wp^{\prime}(\varpi)=0$, while $\wp(\varpi)$ is a real root of $4 w^{3}+4=0$; that is, $\wp(\varpi)=-1$. Thus, since $\wp$ is the inverse of an elliptic integral, we must have

$$
\begin{aligned}
\varpi & =\int_{-1}^{\infty} \frac{d w}{\sqrt{4 w^{3}+4}}=\frac{1}{2} \int_{-1}^{0} \frac{d w}{\sqrt{w^{3}+1}}+\frac{1}{2} \int_{0}^{\infty} \frac{d w}{\sqrt{w^{3}+1}} \\
& =\frac{1}{6} \mathrm{~B}\left(\frac{1}{3}, \frac{1}{2}\right)+\frac{1}{6} \mathrm{~B}\left(\frac{1}{3}, \frac{1}{6}\right)=2^{-7 / 3} 3^{1 / 2} \pi^{-1} \Gamma\left(\frac{1}{3}\right)^{3},
\end{aligned}
$$

as shown by the changes of variables $t=-w^{3}$ and $u=w^{3}$ (respectively) in the last two integrals of the first line, followed by Eulerian Beta function evaluations (12). We henceforth denote the quantity $\varpi$ by $3 r$.

The function $f(i \sqrt{3} z)$ is determined by $f(0)=1$ and by the functional equation deduced from (5):

$$
\frac{1}{i \sqrt{3}} \frac{d}{d z} f(i \sqrt{3} z)=-f(-i \sqrt{3} z)^{2} .
$$

We proceed to verify 23. Since $\wp^{\prime}(3 r)=0$ and $\wp(3 r)=-1$, we first have

$$
\frac{-\wp^{\prime}(3 r)-2 i \sqrt{3}}{2 i \sqrt{3} \wp(3 r)}=f(0)=1 \text {. }
$$

\footnotetext{
2 These are the only rational points of the curve $\mathcal{E}$, since it is known that the Mordell curve $Y^{2}=X^{3}+1$ has six rational points; see, for instance, the SIMATH tables that are accessible at tnt.math.metro-u.ac.jp/simath/MORDELL/MORDELL+.

${ }^{3}$ The reader should be warned that the six numbers $\pm 6 r, \pm 6 r e^{ \pm i \pi / 3}$ are not the shortest nonzero elements of the period lattice for $\wp$. They generate a sublattice of index 3 in the period lattice of $\wp$ whose shortest elements are given by the six numbers $\pm 2 i \sqrt{3} r, \pm 2 i \sqrt{3} r e^{ \pm i \pi / 3}$; see also Section 4 and Figure 1

${ }^{4}$ We have $\wp(\varpi)=0$ since $\wp^{\prime}$ is odd; hence $\wp(\varpi)$ must be a root of the third-degree polynomial associated with $\wp$; here, $\wp(\varpi)=-1$.
} 
In view of (24), the equality (23) then reduces to proving the identity

$$
\frac{1}{i \sqrt{3}}\left(\frac{-\wp^{\prime}(z+3 r)-2 i \sqrt{3}}{2 i \sqrt{3} \wp(z+3 r)}\right)^{\prime}+\left(\frac{-\wp^{\prime}(-z+3 r)-2 i \sqrt{3}}{2 i \sqrt{3} \wp(-z+3 r)}\right)^{2}=0 .
$$

Since $\wp(z)$ is an even function, which is $6 r$-periodic, the left-hand side of 25 can be put under the rational form $A / B$, where the numerator $A$ involves $\wp, \wp^{\prime}, \wp^{\prime \prime}$ at $z+3 r$. Writing $\wp, \wp^{\prime}, \wp^{\prime \prime}$ for $\wp(z+3 r), \wp^{\prime}(z+3 r), \wp^{\prime \prime}(z+3 r)$, it remains to verify that

$$
A=-2 \wp^{\prime \prime} \wp+2\left(\wp^{\prime}+2 i \sqrt{3}\right) \wp^{\prime}+\wp^{\prime 2}-4 i \sqrt{3} \wp^{\prime}-12
$$

vanishes identically. Derivation of the differential equation $\wp^{\prime 2}=4 \wp^{3}+4$ for $\wp$ yields $\wp^{\prime \prime}=6 \wp^{2}$ and we indeed obtain

$$
A=-12 \wp^{3}+3 \wp^{\prime 2}-12=0,
$$

which concludes the proof of the statement.

\section{Lattice-sum expressions for the pseudo-factorials $\alpha_{n}$}

From the Dixonian as well as the Weierstraß connections discussed in the previous section, expressions of the $\alpha_{n}$ as lattice sums can be developed.

Theorem 3. The pseudo-factorials are expressible as lattice sums involving a twelfth root of unity: with $\rho=2 r \sqrt{3}$ and $r$ as in Eq. 22 , one has, for any $n \geq 2$ :

$$
\alpha_{n}=-\frac{n !}{\rho^{n+1}} \sum_{\lambda, \mu \in \mathbb{Z}} \frac{\zeta^{8 \lambda+4 \mu}}{\left[\left(\lambda-\frac{1}{2}\right) \zeta+\left(\mu-\frac{1}{2}\right) \zeta^{-1}\right]^{n+1}}, \quad \zeta:=e^{i \pi / 6} .
$$

The formula (26) implies explicit asymptotics for $\left(\alpha_{n}\right)$, namely,

$$
\frac{\alpha_{2 \nu}}{(2 \nu) !} \sim(-1)^{\nu} 3^{-\nu} r^{-2 \nu-1} \text { and } \frac{\alpha_{2 \nu+1}}{(2 \nu+1) !} \sim(-1)^{\nu+1} 3^{-\nu-1} r^{-2 \nu-2},
$$

once only the relevant dominant poles are retained. This explains in particular the regular sign pattern " +--+ " observed in (2).

Proof. Let $G(z)$ be an elliptic (i.e., meromorphic, doubly periodic) function that has only simple poles. Let $\Lambda=\mathbb{Z} \omega_{1} \oplus \mathbb{Z} \omega_{2}$ be its lattice of periods and let $\mathcal{S}_{0}$ be the set of poles contained in a fundamental domain of $\Lambda$. Then, if $0 \notin \mathcal{S}_{0}$, one has

$$
G(z)=G(0)+z G^{\prime}(0)+\sum_{\omega \in \Lambda} \sum_{\rho \in \mathcal{S}_{0}} r_{\rho}\left[\frac{1}{z-(\rho+\omega)}+\frac{1}{\rho+\omega}+\frac{z}{(\rho+\omega)^{2}}\right]
$$

where $r_{s}$ represents the residue of $G(z)$ at $z=s$. Theorem 2 and the formula (27) show that it suffices, up to an affine transformation, to work out the singular structure of

$$
H(z)=\frac{-\wp^{\prime}(z)-2 i \sqrt{3}}{2 i \sqrt{3} \wp(z)}
$$

in order to deduce the partial fraction expansion of $f(z)$, from which the lattice sum (26) expression will result.

The function $\wp(z) \equiv \wp(z ; 0,-4)$ has lattice of periods

$$
\left(\mathbb{Z} e^{i \pi / 6} \oplus \mathbb{Z} e^{-i \pi / 6}\right) 2 \sqrt{3} r
$$



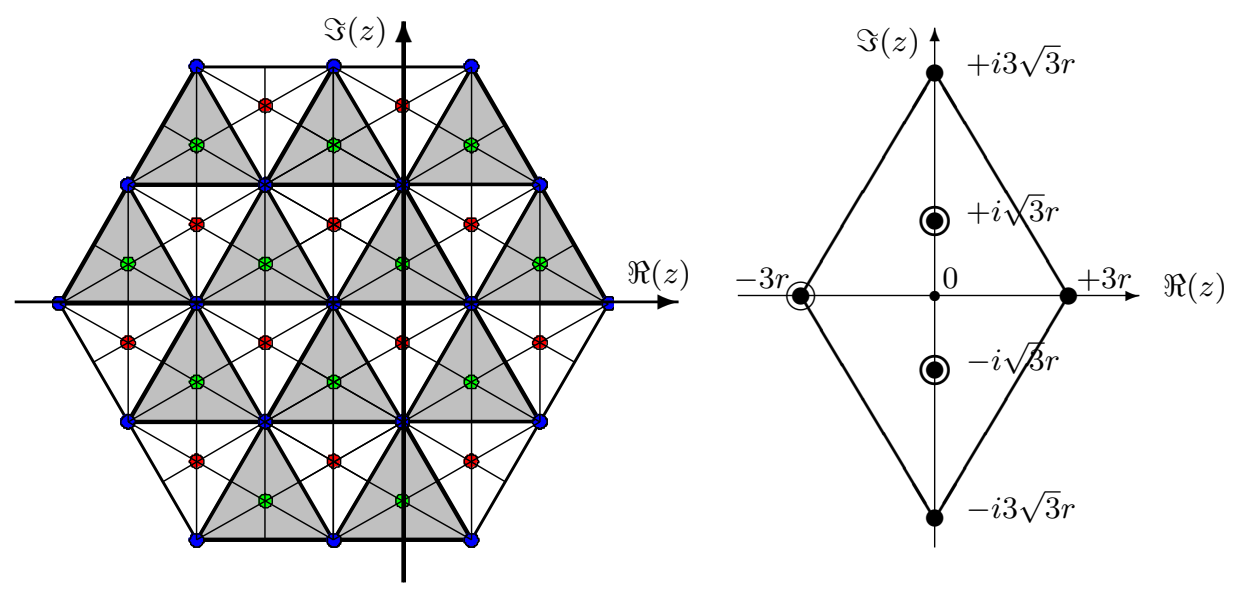

FiguRE 1. Left: the "primary" lattice of periods [thick lines] of $f(z)$, where a fundamental domain is obtained by the union of two adjacent equilateral triangles (one grey, one white); the poles (three per fundamental domain) are represented by small discs and form a "secondary" hexagonal lattice [thin lines]. Right: a diagram showing the three poles (circled) of a fundamental domain of $f(z)$ around the origin.

and $H(z)$ has simple poles at $z=0$ and $z= \pm 2 r$. Given the series expansion $\wp(z)=z^{-2}+O\left(z^{2}\right)$ of $\wp$ around 0 , we find the residue of $H(z)$ at 0 to be

$$
\operatorname{Res}(H(z) ; z=0)=\frac{-i}{\sqrt{3}} \text {. }
$$

By the discussion following (21), we also have $\left(\wp( \pm 2 r), \wp^{\prime}(2 r)\right)=(0, \pm 2)$. Moreover, we have $\left(\wp(3 r), \wp^{\prime}(3 r)\right)=(-1,0)$ and $\left(\wp^{\prime}(3 r), \wp^{\prime \prime}(3 r)\right)=\left(\wp^{\prime}(3 r), 6 \wp(3 r)^{2}\right)=(0,6)$, hence $\wp^{\prime}(2 r)=-2$ and $\wp^{\prime}(4 r)=\wp^{\prime}(-2 r)=2$. The expansion of $\wp(z)$ around $2 r$ is thus

$$
\wp(z+2 r)=-2 z+2 z^{4}-\frac{8}{7} z^{7}+\frac{4}{7} z^{10}+\cdots,
$$

from which a simple computation provides the residue of $H(z)$ at $2 r$, and, similarly, the residue at $-2 r$ :

$$
\operatorname{Res}(H(z) ; z=2 r)=\frac{3+i \sqrt{3}}{6}, \quad \operatorname{Res}(H(z) ; z=-2 r)=\frac{-3+i \sqrt{3}}{6},
$$

Now, by (28), 29), and (30), the singular structure of $H(z)$ is entirely known. Since $f(i z \sqrt{3})=H(z+3 r)$, an affine transformation (composed of a translation, a rotation, and a dilation) provides the singular structure of $f(z)$ itself - we abbreviate the discussion, which is routine. As represented in Figure 1 , the lattice of periods $\Lambda$ of $f(z)$ is a hexagonal lattice with generators $3 r(-1 \pm i \sqrt{3})$; we may call it the "primary" hexagonal lattice. There are three poles of $f(z)$ in the fundamental domain, at $-3 r$ and $\pm i \sqrt{3} r$, which, upon translation by $\Lambda$, generate a "secondary" hexagonal lattice. The residue of $f(z)$ (deduced from $(29)$ and $(30)$ ), is then found to be of the form $\zeta^{8 \lambda+4 \mu}$ at a point of the form $2 r \sqrt{3}\left[(\lambda-1 / 2) \zeta+(\mu-1 / 2) \zeta^{-1}\right]$, which corresponds to a 3 -colouring of the secondary hexagonal lattice (since $\zeta^{4}$ is a 
third root of unity). The corresponding partial fraction decomposition 27) results for $f(z)$. Finally, the fact $5^{5}$ that, for $n \geq 2$,

$$
\left[z^{n}\right]\left(\frac{1}{z-a}+\frac{1}{a}+\frac{z}{a^{2}}\right)=-\frac{1}{a^{n+1}} .
$$

yields the stated lattice-sum formula for $\alpha_{n}$.

The sum (26) establishes the pseudo-factorials as a two-dimensional analogue of Bernoulli and Euler numbers, one that is relative to the hexagonal lattice. It might be of interest to investigate systematically continued fractions relative to other hexagonal lattice sums. It is worthy of note that arithmetic properties of analogous "lemniscatic" sums, relative to the square lattice, have been studied by Hurwitz [10, 11].

\section{The Stieltjes-Rogers addition theorem and continued fractions}

This section serves to introduce the basic technology needed to develop an explicit continued fraction expansion from an addition theorem of a suitable form. An experimental approach specialized to pseudo-factorials follows, in Section6. We can then reap the crop in Section 7 and finally prove our main continued fraction result (Theorem 5).

Stieltjes and Rogers independently discovered that the continued fraction expansion of an ordinary generating function, $\Phi(z)=\sum \phi_{n} z^{n}$, is closely related to addition formulae satisfied by the corresponding exponential generating function, $\phi(z)=\sum \phi_{n} z^{n} / n$ !. First, a definition.

Definition 1. Let $\phi(z)=1+\sum_{n \geq 1} \phi_{n} z^{n} / n$ ! be a formal power series. It is said to satisfy an addition formula of the Stieltjes-Rogers type if there exist nonzero constants $\omega_{1}, \omega_{2}, \ldots$ and formal power series $\varphi_{0}(z), \varphi_{1}(z), \ldots$, such that

$$
\phi(x+y)=\varphi_{0}(x) \varphi_{0}(y)+\omega_{1} \varphi_{1}(x) \varphi_{1}(y)+\omega_{2} \varphi_{2}(x) \varphi_{2}(y)+\cdots,
$$

where $\varphi_{r}(z)$ has valuation $r$ and is normalized by $\varphi_{r}(z)=\left(z^{r} / r !\right)+O\left(z^{r+1}\right)$.

In (31), the valuation condition on $\varphi_{r}$ is essential, the normalization $\phi_{r}(z) \sim$ $z^{r} / r$ ! being a mere convenience for what follows.

The addition formula gives rise to an algorithm for computing the $\varphi_{\ell}(z)$, knowing $\phi(z)$, either symbolically or via some series expansion. First, setting $y=0$ in the addition formula shows that $\phi(z)$ must be equal to $\varphi_{0}(z)$ (this makes use of the normalization $\left.\varphi_{0}(0)=1\right)$. Next, assume that the functions $\varphi_{0}, \ldots, \varphi_{\ell-1}$ and the coefficients $\omega_{1}, \ldots, \omega_{\ell-1}$ have already been determined. Then, by differentiating $\ell$ times the addition formula (31) with respect to $y$, then setting $y=0$, one finds

$$
\partial_{x}^{\ell} \phi(x)-\sum_{j=0}^{\ell-1} \omega_{j} \varphi_{j}(x)\left[\partial_{y}^{\ell} \varphi_{j}(y)\right]_{y=0}=\omega_{\ell} \varphi_{\ell}(x) .
$$

Upon comparing the coefficient of $x^{\ell}$ in the Taylor expansions of both sides, we see that at most one nonzero coefficient $\omega_{\ell}$ can satisfy the equation, given the normalization $\varphi_{\ell}(x)=x^{\ell} / \ell !+O\left(x^{\ell+1}\right)$. Once this choice has been fixed, then $\varphi_{\ell}(x)$

\footnotetext{
${ }^{5}$ Notation: we let $\left[z^{n}\right] f$ represent the coefficient of $z^{n}$ in the formal power series or analytic function $f$.
} 
is uniquely determined as a linear combination of the previous $\varphi_{j}(z)$, together with derivatives of $\phi$, and the process can continue. This construction also determines the broad class of functions in which the $\varphi_{\ell}(z)$ live: each $\varphi_{\ell}(z)$ belongs to the vector space generated over $\mathbb{C}$ by the first derivatives $\phi, \phi^{(1)}, \ldots, \phi^{(\ell)}$ of the function $\phi(z)$. This algorithm, albeit suboptimal from a computational point of view, permits us to experiment with addition formulae relative to the generating function of any given number sequence $\left(\phi_{n}\right)$, a fact that will prove especially valuable in Section 6 .

Addition formulae of the Stieltjes-Rogers type are logically equivalent to continued fraction expansions as expressed by the following central theorem originally due to Stieltjes [29] and Rogers [24]; see also Perron [21, p. 133] and Wall [31, p. 204].

Theorem 4 (Stieltjes-Rogers). Let the exponential generating function $\phi(z)=$ $1+\sum_{n \geq 1} \phi_{n} z^{n} / n$ ! satisfy an addition formula of the form (31). Then, the corresponding ordinary generating function $\Phi(z)=1+\sum_{n \geq 1} \phi_{n} z^{n}$ admits a Jacobi-type continued fraction expansion 6

$$
\Phi(z)=\frac{1}{1-c_{0} z-\frac{a_{1} z^{2}}{1-c_{1} z-\frac{a_{2} z^{2}}{\cdot}}},
$$

where the coefficients are determined by

$$
a_{j}=\frac{\omega_{j}}{\omega_{j-1}} \quad(j \geq 1), \quad c_{j}=\varphi_{j, j+1}-\varphi_{j-1, j} \quad(j \geq 0) .
$$

There, $\varphi_{j, k}=k !\left[z^{k}\right] \varphi_{j}(z), \varphi_{-1, k}=0$, and $\omega_{0}=1$.

As an illustration, following Stieltjes and Rogers, we examine the case of

$$
\phi(z)=\sec (z) \equiv \frac{1}{\cos (z)} .
$$

Each $\varphi_{k}(z)$ (provided it exists) must then a priori be of the form $\sec (z) P_{k}(\tan (z))$, where $P_{k}$ is a polynomial satisfying $\operatorname{deg} P_{k}=k$. In a simple case like this, classical trigonometric identities yield

$$
\sec (x+y)=\frac{1}{\cos (x) \cos (y)-\sin (x) \sin (y)}=\sum_{k \geq 0} \sec (x) \tan (x)^{k} \cdot \sec (y) \tan (y)^{k},
$$

which, in normalized form, becomes

$$
\sec (x+y)=\sum_{k \geq 0}(k !)^{2}\left(\sec (x) \frac{\tan (x)^{k}}{k !}\right) \cdot\left(\sec (y) \frac{\tan (y)^{k}}{k !}\right) .
$$

With $E_{n}=n !\left[z^{n}\right] \sec (z)$ an Euler number, Theorem 4 then provides the continued fraction expansion:

$$
\sum_{n \geq 0} E_{n} z^{n}=\frac{1}{1-\frac{1^{2} \cdot z^{2}}{1-\frac{2^{2} \cdot z^{2}}{\cdot}}}
$$

\footnotetext{
${ }^{6}$ Such an expansion is diversely known as a Jacobi fraction, a $J$-fraction, or an associated continued fraction.
} 
where the coefficients $1^{2}, 2^{2}, 3^{2}, \ldots$, are obtained here as quotients of consecutive squared factorials. The absence of linear terms reflects the parity of $\sec (z)$.

\section{Experimental determination of the addition formula for $f(z)$}

Section 5 has shown that, in order to approach the construction of a continued fraction expansion for pseudo-factorials, we need to develop a suitably constrained addition formula for their exponential generating function $f(z)$, which is elliptic. We proceed here in an experimental manner in order to infer the likely shape of such an addition formula 7 . Once this had been done, the proof of our main continued fraction reduces to purely mechanical verifications to be carried out in the next section.

A first idea that comes to mind is to look for an addition formula of a kind similar to the secant case (33), namely,

$$
\phi(x+y)=\phi(x) \phi(y) \Psi(\sigma(x) \cdot \sigma(y)),
$$

for some function (or power series) $\Psi(w)$. However, all such formulae can only arise from a class of special functions that comprises five parametrized subclasses, of which prototypes are

$$
\sec (z), \quad \frac{1}{1-z}, \quad e^{e^{z}-1}, \quad e^{z^{2} / 2}, \quad \frac{1}{2-e^{z}} .
$$

(This is a rephrasing of a classification of orthogonal polynomial systems due to Meixner [3, 19].) Obviously, elliptic functions are not amongst this group.

Another source of inspiration is a continued fraction relative to elliptic functions, which is also due to Stieltjes and Rogers. With sn, cn, dn the Jacobian elliptic functions, as classically defined, where we leave the modulus $k$ implicit, we have

$$
\operatorname{cn}(x+y)=\frac{\operatorname{cn} x \operatorname{cn} y-\operatorname{sn} x \operatorname{sn} y \operatorname{dn} x \operatorname{dn} y}{1-k^{2} \operatorname{sn}^{2} x \operatorname{sn}^{2} y},
$$

see for instance [32, p. 497]. This can be put into an equivalent Stieltjes-Rogers form (up to normalization), namely,

$\operatorname{cn}(x+y)=\operatorname{cn} x \operatorname{cn} y\left(1-\operatorname{sn} x \operatorname{dn} x \operatorname{sn} y \operatorname{dn} y+k^{2} \operatorname{sn} x \operatorname{cn}^{2} x \operatorname{dn} x \operatorname{sn} y \operatorname{cn}^{2} y \operatorname{dn} y+\cdots\right)$, corresponding to the continued fraction expansion

$$
\sum_{n \geq 0} \mathrm{cn}_{n} z^{n}=\frac{1}{1-\frac{1^{2} \cdot z^{2}}{1-\frac{2^{2} k^{2} \cdot z^{2}}{1-\frac{3^{2} \cdot z^{2}}{\ddots}}}}
$$

where $\mathrm{cn}_{n}:=n !\left[z^{n}\right] \operatorname{cn}(z)$.

We now turn to the continued fraction expansion relative to pseudo-factorials which, by Theorem 4 involves determining the right addition formula for $f(z)$. Based on experiments under the Maple system as well as on induction from the

\footnotetext{
${ }^{7}$ This section is not, strictly speaking, necessary. It could have been replaced by the shorter but somewhat obscure formulation: "Crystal ball gazing revealed to us the addition formula 40,."
} 
secant (33) and Jacobian (35) cases, we started searching for an addition formula of the form

$$
f(x+y)=f(x) f(y) \Psi(\sigma(x) \sigma(y))+h(x) h(y) \Xi(\tau(x) \tau(y)),
$$

for some power series $\Psi, \Xi, h, \sigma, \tau$, with (at least) $\Psi_{0}=\Xi_{0}=1$, and $\sigma(x), \tau(x), h(x)$ all being $O(x)$. Since some binary pattern is present in the continued fraction, it is natural further to suppose that $\sigma(x)=O\left(x^{2}\right), \tau(x)=O\left(x^{2}\right)$, which then corresponds to an "odd-even" addition formula,

$$
f(x+y)=f(x) f(y)+h(x) h(y)+\Psi_{1} f(x) f(y) \sigma(x) \sigma(y)+\Xi_{1} h(x) h(y) \tau(x) \tau(y)+\cdots,
$$

where $\Psi_{m}=\left[w^{m}\right] \Psi(w)$ and $\Xi_{m}=\left[w^{m}\right] \Xi(w)$. In the notations of (31), we thus hope for an addition formula of the form

$$
\varphi_{2 j}(x) \propto f(x) \sigma(x)^{j}, \quad \varphi_{2 j+1}(x) \propto h(x) \tau(x)^{j-1},
$$

where $a(x) \propto b(x)$ means that the ratio $a(x) / b(x)$ is a constant.

The pleasant feature of the conjectured expansions $(37)$ and $\sqrt{39}$ is that their plausibility can be effectively tested. Indeed, from the previous section, we have available an algorithm that can determine the (unique) $\varphi_{j}(x)$ corresponding to $\phi(z) \equiv f(z)$, this to any desired precision. It then suffices to check that

$$
\frac{\varphi_{2}(x)}{\varphi_{0}(x)} \propto \frac{\varphi_{4}(x)}{\varphi_{2}(x)} \propto \cdots, \quad \text { and } \quad \frac{\varphi_{3}(x)}{\varphi_{1}(x)} \propto \frac{\varphi_{5}(x)}{\varphi_{3}(x)} \propto \cdots .
$$

Verification of these relations for about a dozen of the $\varphi_{j}$ and till orders in the range 50-100 convinces us that we are on the right tracks.

In fact, we found experimentally that $\sigma(z)=\tau(z)$ up to $O\left(z^{50}\right)$, the series starting as

$$
\sigma(z)=3\left(z^{2}-z^{4}+z^{6}-\frac{6}{7} z^{8}+\frac{5}{7} z^{10}-\cdots\right) .
$$

Also, the function $h(z)$ that appears in 38 must be proportional to $\varphi_{1}(z)$ of the addition formula, whose expansion starts as $\varphi_{1}(z)=z-z^{3}+\frac{1}{4} z^{4}-\cdots$. That function $\varphi_{1}(z)$ must itself, on general grounds, be a linear combination of $f(z)$ and $f^{\prime}(z)$ without constant term, so that

$$
\varphi_{1}(z)=-\frac{1}{3}\left(f(z)+f^{\prime}(z)\right) \text { and } \quad h(z) \propto \varphi_{1}(z) .
$$

Finally, assuming the observed law of the coefficients in the continued fraction to hold forever, we can deduce the only possible shape of the $\Xi$ and $\Psi$ functions. The function $\sigma$ is then inferred on the basis of the fact that $\varphi_{2}(z) \propto \varphi_{0}(z) \sigma(z)$ must be a linear combination of $f, f^{\prime}, f^{\prime \prime}$. All in all, every ingredient of an addition formula of type (37) is in place, and we are eventually led to conjecturing an addition formula for $f(z)$

$$
\left\{\begin{array}{l}
f(x+y)=\frac{f(x) f(y)-\frac{1}{3} h(x) h(y)}{1-\frac{1}{3} \sigma(x) \sigma(y)} \\
h(z)=f(z)+f^{\prime}(z), \quad \sigma(z)=1-f(z) f(-z),
\end{array}\right.
$$

which we shall establish in the next section. 


\section{Proof of the continued fraction expansion}

At this stage, we know that establishing the continued fraction (4) relative to the ordinary generating function $F(z)$ of pseudo-factorials reduces to deciding the validity of the conjectured addition formula 40 for the exponential generating function $f(z)$. The proof we propose is a computer-assisted verification. As we shall explain, it only involves routine algebraic manipulations 8 namely, rational function operations, normalizations, substitutions, as well as multivariate polynomial divisions. The calculations were performed using the MAPLE computer algebra engine (version 11). Without any attempt at optimization (we purposely wanted our program to rely solely on the most basic algebraic operations), the mechanical verification reduces to the mere execution of a few billion machine instructionscurrently, just a few seconds of elapsed time.

Proposition 1. The function $f(z)$ satisfies the following addition formula:

$$
f(x+y)=\frac{f(x) f(y)-\frac{1}{3}\left(f(x)+f^{\prime}(x)\right)\left(f(y)+f^{\prime}(y)\right)}{1-\frac{1}{3}(1-f(x) f(-x))(1-f(y) f(-y))} .
$$

Proof. We can a priori appeal to either the Dixon or the Weierstraß framework, and we have opted for the latter. The Weierstraß $\wp$-function, $\wp(z) \equiv \wp(z ; 0,-4)$ satisfies the two algebraic relations

$$
\begin{aligned}
& \wp^{\prime}(z)^{2}=4 \wp(z)^{3}+4 \\
& \wp(u+v)=\frac{1}{4}\left(\frac{\wp^{\prime}(u)-\wp^{\prime}(v)}{\wp(u)-\wp(v)}\right)^{2}-\wp(u)-\wp(v)
\end{aligned}
$$

The first one (DEF) is the basic differential equation, which serves as definition of $\wp$; the second one (ADD) is the familiar addition theorem of elliptic function theory [32, p. 441]. Both are "known" to MAPLE; both can be viewed as deterministic rewrite rules permitting one to expand and simplify expressions involving $\wp$.

Let $6 r$ be the fundamental constant (real period) of Section 3 . We know that $\left(\wp(3 r), \wp^{\prime}(3 r)\right)=(-1,0)$. The addition rule (ADD) combined with the expression of $f(z)$ stated in Theorem 2, Equation (23), then mechanically expresses $f(z)$ as a rational fraction in $\wp(Z)$ and $\wp^{\prime}(Z)$, where $Z=z / i \sqrt{3}$. Similar expressions are obtained for $f^{\prime}(z)$ (by standard derivation rules combined with partial reductions by $(\mathrm{DEF})$ ) and $f(-z)$ (since $\wp$ is an even function, while $\wp^{\prime}$ is odd). In this way, one automatically obtains rational forms in $\wp, \wp^{\prime}$ for

$$
f(x), f^{\prime}(x), f(y), f^{\prime}(y), f(-x), f(-y), f(x+y),
$$

where the last one necessitates a substitution $z \mapsto x+y$, followed by an application of the $(\mathrm{ADD})$ rule.

Let now $\mathcal{D}$ be the difference between the left-hand side and the right-hand side of the relation to be proved, Equation (41). By the process described above, $\mathcal{D}$ becomes a rational function, with coefficients in $\mathbb{Q}(i \sqrt{3})$, in the four quantities $X_{1}, Y_{1}, X_{2}, Y_{2}$, where $X_{1}=\wp(x / i \sqrt{3}), Y_{1}=\wp^{\prime}(x / i \sqrt{3})$, and similarly for $X_{2}, Y_{2}$, with $y$ replacing $x$. The (rather large) rational fraction normalizes to the form

\footnotetext{
${ }^{8}$ The validity of intermediate steps is, in addition, easily cross-checked by means of Taylor series expansions.
} 
$\mathcal{D}=A / B$, with numerator $A$ and denominator $B$ involving, respectively, 2388 and 1256 monomials. One can then operate with the rule (DEF), instantiated as

$$
Y_{1}^{2} \mapsto 4 X_{1}^{3}+4, \quad Y_{2}^{2} \mapsto 4 X_{2}^{3}+4,
$$

the corresponding reductions being simply effected by multivariate polynomial divisions. When this is done, we find that $A$ reduces to 0 , while $B$ is reduced to a nonzero polynomial, which is of degree 1 in $Y_{1}, Y_{2}$ and of degree 8 in $X_{1}, X_{2}$. The verification of the addition formula for $f(z)$ is thereby completed.

A direct application of Theorem 4 to the addition formula expressed by Proposition 1 gives rise to our main continued fraction.

Theorem 5. The ordinary generating function of the pseudo-factorials satisfies

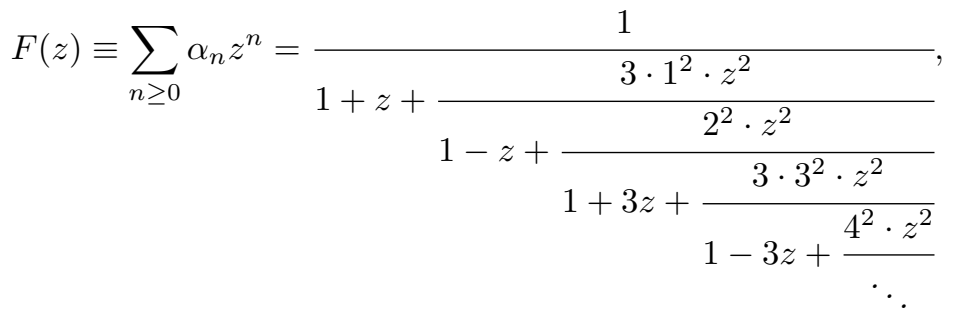

where the coefficients are, with the notations of the Jacobi form (32):

$$
c_{j}=(-1)^{j-1}\left(j+\frac{1+(-1)^{j}}{2}\right), \quad a_{j}=-j^{2}\left(2-(-1)^{j}\right) .
$$

Proof. We make use of the addition formula [41], which, taken under the form 40], yields

$$
f(x+y)=\sum_{n=0}^{\infty} \omega_{2 n} \varphi_{2 n}(x) \varphi_{2 n}(y)+\sum_{n=0}^{\infty} \omega_{2 n+1} \varphi_{2 n+1}(x) \varphi_{2 n+1}(y),
$$

with the $\omega_{n}$ and $\varphi_{n}$ determined by

$$
\begin{cases}\omega_{2 n} \varphi_{2 n}(x) \varphi_{2 n}(y) & =+3^{-n} f(x) f(y) \sigma(x)^{n} \sigma(y)^{n} \\ \omega_{2 n+1} \varphi_{2 n+1}(x) \varphi_{2 n+1}(y) & =-3^{-n-1} h(x) h(y) \sigma(x)^{n} \sigma(y)^{n}\end{cases}
$$

We have, with the notations of (40), $f(z)=1-z+O\left(z^{2}\right)$, as well as $\sigma(z) \equiv$ $1-f(z) f(-z)=3 z^{2}+O\left(z^{4}\right)$ and $h(z) \equiv f(z)+f^{\prime}(z)=-3 z+O\left(z^{3}\right)$. The required normalization of a Stieltjes-Rogers addition formula, $\varphi_{n}(z)=z^{n} / n !+O\left(z^{n+1}\right)$, combined with the low-order expansions of $f(z), h(z), \sigma(z)$, gives us

$$
\omega_{2 n}=3^{n}(2 n) !^{2}, \quad \omega_{2 n+1}=-3^{n+1}(2 n+1) !^{2},
$$

as well as

$$
\varphi_{2 n}(z)=\frac{z^{2 n}}{(2 n) !}-(2 n+1) \frac{z^{2 n+1}}{(2 n+1) !}+O\left(z^{2 n+2}\right), \quad \varphi_{2 n+1}(z)=\frac{z^{2 n+1}}{(2 n+1) !}+O\left(z^{2 n+3}\right) .
$$

We thus have

$$
\frac{\omega_{1}}{\omega_{0}}=-3, \quad \frac{\omega_{2}}{\omega_{1}}=-2^{2}, \frac{\omega_{3}}{\omega_{2}}=-3 \cdot 3^{2}, \quad \frac{\omega_{4}}{\omega_{3}}=-4^{2}, \cdots,
$$

and, for $c_{j}:=\varphi_{j, j+1}-\varphi_{j-1, j}$ :

$$
c_{0}=-1, \quad c_{1}=1, \quad c_{2}=-3, \quad c_{3}=3, \quad c_{4}=-5, \quad c_{5}=5, \cdots .
$$

By Theorem 4, the last two formulae conclude the proof of 42 . 


\section{A family of orthogonal polynomials}

Our goal in this section consists in finding an explicit form for the polynomials that appear in the convergents of the main continued fraction of Theorem 5 , this by way of their exponential generating function. We focus our attention on the denominator polynomials, precisely, on their reciprocals, which form a family of formally orthogonal polynomials that appears to be new.

We start by specializing to the continued fraction under consideration 42 some well-known algebraic properties found in [21, 31] that hold for an arbitrary Jacobi fraction (32). The convergents of 42 are obtained by truncating the infinite fraction before a numerator. In this way, a collection of rational fractions $P_{k}(z) / Q_{k}(z)$ of increasing degrees is obtained,

$$
\frac{0}{1}, \quad \frac{1}{1+z}, \quad \frac{1-z}{1+2 z^{2}}, \quad \frac{1+2 z+z^{2}}{1+3 z+6 z^{2}+10 z^{3}}, \quad \frac{1-z+22 z^{2}-30 z^{3}}{1+24 z^{2}-8 z^{3}+24 z^{4}},
$$

so that $Q_{0}=1, Q_{1}=1+z$, and so on. The denominator polynomials $Q_{k}$ satisfy a "three-term recurrence" relation,

$$
Q_{k}=\left(1-c_{k-1} z\right) Q_{k-1}-a_{k-1} z^{2} Q_{k-2} .
$$

(The $P_{k}$ satisfy the same recurrence, but with initial conditions $P_{0}=0, P_{1}=1$.) The reciprocal polynomials defined by

$$
q_{k}(z)=z^{k} Q_{k}\left(\frac{1}{z}\right)
$$

then satisfy the recurrence

$$
q_{k}=\left(z-c_{k-1}\right) q_{k-1}-a_{k-1} q_{k-2}, \quad q_{-1}=0, \quad q_{0}=1,
$$

with the $a_{j}, c_{j}$ as in (43). On general grounds, they are formally orthogonal with respect to a (formal) measure whose moments coincide with the pseudofactorials, $\left(\alpha_{n}\right)$. In other words, they are orthogonal with respect to the bilinear form

$$
\langle f, g\rangle=\langle f g\rangle, \quad \text { with } \quad\left\langle z^{n}\right\rangle=\alpha_{n} .
$$

Observe finally that, once the $Q_{k}$ are known, the $P_{k}$ can somehow be regarded as known. Indeed, relative to (32), one has, in the sense of formal power series,

$$
Q_{k}(z) F(z)-P_{k}(z)=O\left(z^{2 k}\right),
$$

so that the coefficients of the $P_{k}$ are expressible as a convolution of the two sequences $\left[z^{n}\right] Q_{k}(z)$ and $\alpha_{n} \equiv\left[z^{n}\right] F(z)$.

We have the following characterization.

Theorem 6. Let $\Upsilon(z, t)$ be the exponential generating function of the reciprocal polynomials $\left(q_{k}\right)$ of (45), with coefficients 43]:

$$
\Upsilon(z, t):=\sum_{k=0}^{\infty} q_{k}(z) \frac{t^{k}}{k !} .
$$

Consider the algebraic curve

$$
2+3 t+3 t(1+t) \eta-2\left(1-3 t^{2}+3 t^{4}\right) \eta^{3}=0,
$$


which is of genus 0 and is parametrized by

$$
t=\frac{1}{3} \frac{\left(w^{2}+3\right) w}{w^{2}+1}, \quad \eta=3 \frac{(w+1)\left(w^{2}+1\right)}{w^{4}+3},
$$

and let $\eta(t)$ be the branch that satisfies $\eta(0)=1$ :

$$
\eta(t)=1+t+2 \frac{t^{2}}{2 !}+10 \frac{t^{3}}{3 !}+24 \frac{t^{4}}{4 !}+280 \frac{t^{5}}{5 !}+400 \frac{t^{6}}{6 !}+12880 \frac{t^{7}}{7 !}-\cdots .
$$

Define

$$
\chi(t):=\sqrt{\eta(t)^{2}-\frac{2 t(1+t)}{1-3 t^{2}+3 t^{4}}}=1+\frac{t^{2}}{2 !}-2 \frac{t^{3}}{3 !}+\frac{t^{4}}{4 !}-100 \frac{t^{5}}{5 !}-575 \frac{t^{6}}{6 !}-\cdots
$$

and introduce the fundamental elliptic integral

$$
J(t):=\int_{0}^{t} \frac{d u}{\sqrt{1-3 u^{2}+3 u^{4}}}=t+3 \frac{t^{3}}{3 !}+45 \frac{t^{5}}{5 !}+1215 \frac{t^{7}}{7 !}+8505 \frac{t^{9}}{9 !}-\cdots
$$

Then, the generating function $\Upsilon$ satisfies

$$
\Upsilon(z, t)=\eta(t) \cosh (z J(t))+\chi(t) \sinh (z J(t)) .
$$

Equation (51) was first arrived at by a combination of induction and of partly heuristic calculations, based on "guessing" intermediate differential equations as well as on MAPLE's symbolic integration capabilities. Rather than offering a heavy proof by successive transformations of the defining recurrence (46), we have opted to present a computer-assisted verification of (51). In this way, we feel we save symbols, hence pages, hence trees. The price to be paid was only a few hours of interaction with the symbolic engine and (eventually!) a few seconds of computer processing time. As in the previous section, only well-specified totally-algorithmic steps are eventually used. Once more, there is no difficulty in checking intermediate steps against series expansions up to order 100 and beyond.

Our proof of the identity (51) eventually boils down to exhibiting a fourth-order differential operator in $t$, with coefficients in $\mathbb{C}(z)$, that is satisfied by the difference between the two sides of (51). It is then sufficient to check that both sides satisfy the same initial conditions given by the coefficients of $t^{0}$ up to $t^{3}$.

The entire process relies on the holonomic framework pioneered by Zeilberger [33, with supporting theorems to be found in works of Stanley, such as [27] and [28, Ch. 6]. Let $\mathbb{K}$ be a ground field, which we take here to be $\mathbb{C}(z)$, the field of rational fractions in $z$. (Throughout, we treat the quantity $z$ as a parameter.) A formal power series of $\mathbb{K} \llbracket t \rrbracket$, simply called "function", is holonomic (alternative names are differentiably finite, $D$-finite, $\partial$-finite) if it satisfies a linear differential equation with coefficients in the rational field $\mathbb{K}(t)$. Equivalently, $h$ is holonomic if the vector space over $\mathbb{K}(t)$ spanned by all the derivatives $\left\{\partial_{t}^{j} h\right\}$ is finite-dimensional. Holonomic functions are known to be closed under sum, product, differentiation, integration, and algebraic substitutions (i.e., substitutions of algebraic functions in place of variables). Finally, if $h$ is holonomic, its sequence of coefficients $\left(\left[z^{n}\right] h\right)$ satisfies a linear recurrence relation with coefficients in $\mathbb{K}(n)$.

Clearly, a holonomic function is determined by a finite amount of information; namely, a defining differential equation supplemented by sufficiently many initial conditions. Given two holonomic functions $A, B$, one can then verify their conjectured identity as follows. 
(i) Compute a differential equation, of order $\omega$, say, that is satisfied by the difference $A-B$.

(ii) Check the coincidence of the expansions of $A$ and $B$ up to terms of order $O\left(t^{\omega}\right)$.

By the finiteness of the underlying vector spaces, the process constitutes a valid proof of $A=B$, in "non-singular" cases at least 19 . In our context, it could be carried out comparatively easily, thanks to the powerful Gfun library developed by Salvy and Zimmermann [25].

Proof (Theorem [6). In what follows, we use $\partial \equiv \partial_{t}$ to represent the differential operator $\frac{\partial}{\partial t}$; we denote by $\mathbf{S} \equiv \mathbf{S}_{n}$ the shift operator on infinite sequences $\left(u_{n}\right)$ such that $\mathbf{S}\left(u_{n}\right)=u_{n+1}$. We let $\Pi_{r}$ generically represent a polynomial of degree $r$, either in $t$ (for differential operators) or in $n$ (for difference operators), with coefficients in $\mathbb{K}$. As indicated before, the quantity $z$ is treated as a parameter. Our purpose is to prove $A=B$, where $A$ is the left-hand side of (51) and $B$ is the right-hand side:

$$
A \stackrel{?}{=} B, \quad \text { with } A:=\Upsilon(z, t), B:=\eta(t) \cosh (z J(t))+\chi(t) \sinh (z J(t)),
$$

and $\eta(t), \chi(t), J(t)$ as defined in the statement. See Figure 2 for a summary of the main steps of our proof.

The left-hand side $(A)$. The parity inherent in the coefficients 43 suggests to introduce the subsequences $r_{n}=q_{2 n}$ and $s_{n}=q_{2 n+1}$. The basic recurrence (46) then relates $r_{n}$ to $r_{n-1}, s_{n-1}$ and $s_{n}$ to $r_{n}, s_{n-1}$; hence, by substitution, the fact that the vector $\left(r_{n}, s_{n}\right)$ depends linearly on $\left(r_{n-1}, s_{n-1}\right)$ via a matrix, whose coefficients are polynomial in $n$ (and the parameter $z$ ). By instantiating this last relation at $n+1$ and $n+2$, and using back substitution, there results that $r_{n}$ and $s_{n}$ satisfy explicit linear recurrences of order 2 with coefficients that are polynomial in $n$. The difference operators annihilating $\left(r_{n}\right)$ and $\left(s_{n}\right)$ are found in this way to be of the form

$$
\Pi_{1} \mathbf{S}^{2}+\Pi_{3} \mathbf{S}^{1}+\Pi_{5} \mathbf{S}^{0} .
$$

Equivalently, the exponential generating functions

$$
R(t)=\sum r_{n} \frac{t^{2 n}}{(2 n) !} \quad \text { and } \quad S(t)=\sum r_{n} \frac{t^{2 n+1}}{(2 n+1) !}
$$

are found to satisfy $\mathfrak{L}[R]=0$ and $\mathfrak{M}[S]=0$, where $\mathfrak{L}$ and $\mathfrak{M}$ are each of the form

$$
\Pi_{5} \partial^{5}+\Pi_{4} \partial^{4}+\cdots+\Pi_{0} \partial^{0} .
$$

In fact, second-order operators $\mathfrak{L}^{\circ}$ and $\mathfrak{M}^{\circ}$ that appear to cancel $R$ and $S$, respectively, can be guessed (roughly, by the method of indeterminate coefficients cleverly implemented in MAPLE's Gfun). The guesses can then be turned into full-fledged proofs by checking (with MAPLE's Ore_algebra, see 4]) the operator divisibility relations: $\mathfrak{L}^{\circ} \mid \mathfrak{L}$ and $\mathfrak{M}^{\circ} \mid \mathfrak{M}$. Once this is done, a differential operator that annihilates $\Upsilon$ can be obtained by making use of properties of holonomic functions

\footnotetext{
${ }^{9}$ An operator is said to be "non-singular" if the lead polynomial of the associated recurrence (relating the coefficients $h_{n}:=\left[t^{n}\right] h(t)$, of a solution $h(t)$ and having coefficients in $\mathbb{K}[n]$ ) has no root in $\mathbb{Z}_{\geq 0}$. In the case of a non-singular operator of order $\omega$, the number of needed initial conditions equals $\omega$. (In the "singular" case, a higher, but still effectively computable, number may be needed.)
} 


\begin{tabular}{ll}
\hline$A \equiv \Upsilon(z, t):$ & $\Pi_{6} \partial^{2} Y+\Pi_{5} \partial Y+\Pi_{4} Y=0 ;$ see $\mathfrak{A}$ in 53 \\
$\eta(t), \chi(t):$ & $\Pi_{6} \partial^{2} Y+\Pi_{5} \partial Y+\Pi_{4} Y=0 ; \eta, \chi$ algebraic 477, 49 \\
$\left\{\begin{array}{c}\exp ( \pm z J(t)) \\
\cosh , \sinh (z J(t))\end{array}\right\}:$ & $\Pi_{4} \partial^{2} Y+\Pi_{3} \partial Y-z^{2} Y=0 ;$ see 454 \\
$\left\{\begin{array}{c}B^{+} \equiv \eta(t) \cosh (z J(t)) \\
B^{-} \equiv \chi(t) \sinh (z J(t))\end{array}\right\}:$ & $\Pi_{12} \partial^{4} Y+\cdots+\Pi_{8} Y=0 ;$ closure algorithm (×) \\
$\Delta:=A-\left(B^{+}+B^{-}\right)$ & $\Pi_{12} \partial^{4} Y+\cdots+\Pi_{8} Y=0 ;$ closure algorithm (+)
\end{tabular}

FIGURE 2. The shape of the differential equations satisfied by quantities intervening in the proof of the main equation (51).

(effective closure under sum) and a further round of simplification based on guessing. It is found in this way that the second-order operator ${ }^{10}$

(53)

$$
\begin{aligned}
\mathfrak{A}= & 4\left(1-3 t^{2}+3 t^{4}\right)\left((2 t(t+1) \zeta-1) \partial^{2}\right. \\
& +4\left(24 t^{5} \zeta+30 t^{4} \zeta-(18+6 \zeta) t^{3}-12 t^{2} \zeta-(4 \zeta-9) t-2 \zeta\right) \partial^{1} \\
& +\left(48 t^{4} \zeta+72 t^{3} \zeta-\left(48+8 \zeta^{3}-18 \zeta\right) t^{2}-\left(6 \zeta+8 \zeta^{3}+12\right) t+9+16 \zeta+12 \zeta^{2}\right) \partial^{0},
\end{aligned}
$$

with $\zeta:=z-1 / 2$, annihilates $\Upsilon(z, t)$.

The right-hand side $(B)$. We can build up differential equations starting with the explicit expression of $B$ in (52): see again Figure 2 for a summary. Given a quantity $X$ that depends on $z$, we set $X^{+}=\frac{1}{2} X(z)+\frac{1}{2} X(-z)$ and $X^{-}=\frac{1}{2} X(z)-\frac{1}{2} X(-z)$, defining its "odd" and "even" parts (in $z$ ), respectively. With $B \equiv \Upsilon(z, t)$, we then consider $B^{+}=\Upsilon^{+}=\eta(t) \cosh (z J(t))$ and $B^{-}=\Upsilon^{-}=\chi(t) \sinh (z J(t))$, and proceed to construct the corresponding annihilators, $\mathfrak{B}^{+}$and $\mathfrak{B}^{-}$.

First, we observe that if $P(t)$ is an arbitrary polynomial, then

$$
Y(t):=\exp \left(z \int^{t} \frac{d w}{\sqrt{P(w)}}\right) \text { satisfies } P \partial^{2} Y+\frac{1}{2} P^{\prime} \partial Y-z^{2} Y=0 .
$$

This equation is invariant by $z \leftrightarrow-z$, so that it is also satisfied when the exponential in (54) is replaced by $\sinh$, cosh.

Next, the function $\eta(t)$, given by a cubic algebraic equation, is found to satisfy a second-order differential equation with coefficients that are of degree at most 6 . The application of closure rules for products of holonomic functions then provides for $B^{+} \equiv \Upsilon^{+}$a differential operator $\mathfrak{B}^{+}$that is of order 4 , with coefficients of degree at most 12 .

We can then proceed to construct the annihilator $\mathfrak{B}^{-}$of the odd part $B^{-} \equiv \Upsilon^{-}$. It turns out that the algebraic function $\chi(t)$ defined in 49 satisfies the same differential equation as $\eta(t)$, but with different initial conditions $\left(\chi(0)=1, \chi^{\prime}(0)=\right.$ $0)$. There now results from this fact and the comments accompanying (54) that we can take

$$
\mathfrak{B}=\mathfrak{B}^{+}=\mathfrak{B}^{-},
$$

10 Much to our surprise, MAPLE's symbolic integrator proposed a solution to $\mathfrak{A}[f]=0$, which involved terms of the rough form $\exp ( \pm z J(t))$ and eventually led us to infer 51. 
as annihilator of the right-hand side $(B)$ of 51 .

The comparison. Finally, it remains to verify that $A=B$. The operator $\Delta$ is defined to annihilate $\mathbf{A}+\mathbf{B}$, where $\mathbf{A}$ and $\mathbf{B}$ are the vector spaces of solutions of $\mathfrak{A}[f]=0$ and $\mathfrak{B}[f]=0$, respectively. By construction, the difference $A-B$ is such that $\Delta[A-B]=0$. The operator, obtained by holonomic closure under sums, is of type

$$
\Delta=\Pi_{12} \partial^{4}+\Pi_{11} \partial^{3}+\Pi_{10} \partial^{2}+\Pi_{9} \partial^{1}+\Pi_{8} \partial^{0} .
$$

The associated recurrence operator is found to be of the form $\Pi_{4} \mathbf{S}^{12}+\cdots+\Pi_{4} \mathbf{S}^{0}$, with leading coefficient

$$
(n+9)(n+10)(n+11)(n+12)\left(9+4 z^{2}\right),
$$

so that $\Delta$ is non-singular at 0 . It thus suffices to verify that the expansions of $A \equiv \Upsilon(z, t)$ and of the right-hand size $B$ in (51) coincide till terms of order $O\left(t^{4}\right)$,

$$
A, B=1+(z+1) t+\left(z^{3}+3 z^{2}+6 z+10\right) \frac{t^{2}}{2 !}+\left(z^{4}+24 z^{2}-8 z+24\right) \frac{t^{3}}{3 !}+O\left(t^{4}\right),
$$

so as to complete the proof that $A=B$. Equation (51) is now established.

Orthogonal polynomials attached to continued fraction expansions relative to elliptic functions, have been first studied by Carlitz and Al-Salam (see [13, 14, for some more recent developments), and, as already mentioned, they form the subject of the monograph Elliptic Polynomials by Lomont and Brillhart [17. The family made explicit by Theorem 6 does not appear to be captured by their classification and hence seems to be new. Remarkably, in connection with birth-and-death processes having cubic weights, Gilewicz et al. 9] have recently discovered another new family of orthogonal polynomials, related to the expansion of the Dixonian function sm taken at 0 (as in [6]), rather than at the point $\pi_{3} / 6$ that is needed here (cf Theorem 1).

\section{Consequences of the continued fraction expansion}

The continued fraction of Theorem 5 has several interesting by-products that we now examine. These include an explicit evaluation of Hankel determinants, as well as elementary congruence properties of pseudo-factorials.

Hankel determinants. It is well known that, generally, coefficients of a Jacobi fraction can be expressed as determinants. This fact is classically derived from Stieltjes's matrix version of the addition theorem [31, pp. 202-206]; it is equivalent to the $L D U$ decomposition of the Gram matrix $H$, with entries $h_{i, j}=\left\langle z^{i}, z^{j}\right\rangle \equiv$ $\left\langle z^{i+j}\right\rangle$ (for $i, j \geq 0$ ), which is also known as the Hankel matrix of the sequence $\left\langle z^{n}\right\rangle$; see for instance [12, §2.1]. Conversely, any known continued fraction yields an explicit Hankel determinant evaluation. Given this, an immediate consequence of Theorem 5 is the following.

Corollary 1. Let $m$ be a positive integer. The Hankel determinant of pseudofactorials

$$
H_{m}^{(0)}:=\left|\begin{array}{cccc}
\alpha_{0} & \alpha_{1} & \cdots & \alpha_{m-1} \\
\alpha_{1} & \alpha_{2} & \cdots & \alpha_{m} \\
\vdots & \vdots & \ddots & \vdots \\
\alpha_{m-1} & \alpha_{m} & \cdots & \alpha_{2 m-2}
\end{array}\right|
$$




\begin{tabular}{r|rrrrrrrrrrrrrrrrrrrrrrrrrrrrr}
\hline \hline$M$ & $n=$ & 0 & 1 & 2 & 3 & 4 & 5 & 6 & 7 & 8 & 9 & 10 & 11 & 12 & 13 & 14 & 15 & 16 & 17 & 18 & 19 & 20 & 21 & 22 & 23 & 24 & 25 \\
\hline 2 & 1 & 1 & 0 & 0 & 0 & 0 & 0 & 0 & 0 & 0 & 0 & 0 & 0 & 0 & 0 & 0 & 0 & 0 & 0 & 0 & 0 & 0 & 0 & 0 & 0 & 0 \\
3 & 1 & 2 & 1 & 2 & 1 & 2 & 1 & 2 & 1 & 2 & 1 & 2 & 1 & 2 & 1 & 2 & 1 & 2 & 1 & 2 & 1 & 2 & 1 & 2 & 1 & 2 \\
4 & 1 & 3 & 2 & 2 & 0 & 0 & 0 & 0 & 0 & 0 & 0 & 0 & 0 & 0 & 0 & 0 & 0 & 0 & 0 & 0 & 0 & 0 & 0 & 0 & 0 & 0 \\
5 & 1 & 4 & 3 & 2 & 1 & 0 & 0 & 0 & 0 & 0 & 0 & 0 & 0 & 0 & 0 & 0 & 0 & 0 & 0 & 0 & 0 & 0 & 0 & 0 & 0 & 0 \\
6 & 1 & 5 & 4 & 2 & 4 & 2 & 4 & 2 & 4 & 2 & 4 & 2 & 4 & 2 & 4 & 2 & 4 & 2 & 4 & 2 & 4 & 2 & 4 & 2 & 4 & 2 \\
7 & 1 & 6 & 5 & 2 & 2 & 2 & 2 & 4 & 1 & 6 & 6 & 6 & 6 & 5 & 3 & 4 & 4 & 4 & 4 & 1 & 2 & 5 & 5 & 5 & 5 & 3 \\
8 & 1 & 7 & 6 & 2 & 0 & 0 & 0 & 0 & 0 & 0 & 0 & 0 & 0 & 0 & 0 & 0 & 0 & 0 & 0 & 0 & 0 & 0 & 0 & 0 & 0 & 0 \\
9 & 1 & 8 & 7 & 2 & 7 & 5 & 4 & 5 & 1 & 8 & 1 & 2 & 7 & 2 & 4 & 5 & 4 & 8 & 1 & 8 & 7 & 2 & 7 & 5 & 4 & 5 \\
10 & 1 & 9 & 8 & 2 & 6 & 0 & 0 & 0 & 0 & 0 & 0 & 0 & 0 & 0 & 0 & 0 & 0 & 0 & 0 & 0 & 0 & 0 & 0 & 0 & 0 & 0 \\
11 & 1 & 1 & 9 & 2 & 5 & 4 & 10 & 6 & 5 & 1 & 1 & 0 & 0 & 0 & 0 & 0 & 0 & 0 & 0 & 0 & 0 & 0 & 0 & 0 & 0 & 0 \\
12 & 1 & 11 & 10 & 2 & 4 & 8 & 4 & 8 & 4 & 8 & 4 & 8 & 4 & 8 & 4 & 8 & 4 & 8 & 4 & 8 & 4 & 8 & 4 & 8 & 4 & 8 \\
13 & 1 & 12 & 11 & 2 & 3 & 12 & 5 & 0 & 5 & 1 & 4 & 2 & 1 & 11 & 9 & 4 & 6 & 11 & 10 & 0 & 10 & 2 & 8 & 4 & 2 & 9 \\
14 & 1 & 13 & 12 & 2 & 2 & 2 & 2 & 4 & 8 & 6 & 6 & 6 & 6 & 12 & 10 & 4 & 4 & 4 & 4 & 8 & 2 & 12 & 12 & 12 & 12 & 10 \\
15 & 1 & 14 & 13 & 2 & 1 & 5 & 10 & 5 & 10 & 5 & 10 & 5 & 10 & 5 & 10 & 5 & 10 & 5 & 10 & 5 & 10 & 5 & 10 & 5 & 10 & 5 \\
16 & 1 & 15 & 14 & 2 & 0 & 8 & 0 & 0 & 0 & 0 & 0 & 0 & 0 & 0 & 0 & 0 & 0 & 0 & 0 & 0 & 0 & 0 & 0 & 0 & 0 & 0 \\
17 & 1 & 16 & 15 & 2 & 16 & 11 & 3 & 3 & 5 & 16 & 7 & 12 & 10 & 7 & 1 & 10 & 1 & 0 & 0 & 0 & 0 & 0 & 0 & 0 & 0 & 0 \\
18 & 1 & 17 & 16 & 2 & 16 & 14 & 4 & 14 & 10 & 8 & 10 & 2 & 16 & 2 & 4 & 14 & 4 & 8 & 10 & 8 & 16 & 2 & 16 & 14 & 4 & 14 \\
19 & 1 & 18 & 17 & 2 & 16 & 17 & 3 & 14 & 0 & 17 & 6 & 9 & 8 & 3 & 18 & 0 & 15 & 8 & 7 & 11 & 3 & 16 & 14 & 3 & 5 & 17 \\
20 & 1 & 19 & 18 & 2 & 16 & 0 & 0 & 0 & 0 & 0 & 0 & 0 & 0 & 0 & 0 & 0 & 0 & 0 & 0 & 0 & 0 & 0 & 0 & 0 & 0 & 0 \\
\hline \hline
\end{tabular}

FiguRE 3. The congruences $\left(\alpha_{n} \bmod M\right)$ for $M=2, \ldots, 20$ and $n=0, \ldots, 25$.

admits the closed form

$$
H_{m}^{(0)}=\prod_{j=1}^{m-1} a_{j}^{m-j}= \begin{cases}(-1)^{m / 2} 3^{m^{2} / 4}\left(\prod_{k=1}^{m-1} k !\right)^{2} & \text { (m even) } \\ (-1)^{(m-1) / 2} 3^{\left(m^{2}-1\right) / 4}\left(\prod_{k=1}^{m-1} k !\right)^{2} & (m \text { odd }),\end{cases}
$$

where the $a_{j}=-j^{2}\left(2-(-1)^{j}\right)$ are the continued fraction numerators of 43 .

Congruences. A cursory examination of the $\alpha_{n}$ suggests clear divisibility patterns; for instance, from (2), we immediately expect the $\alpha_{n}$ to be divisible by 10, for $n$ large enough. Figure 3 tabulates arithmetic congruence properties of the $\alpha_{n}$ for small values of the modulus $M$ and of the index $n$. The table obviously has much structure: the sequence $\left(\alpha_{n}\right)$ appears to be eventually 0 modulo the numbers $2,4,5,8,10,11,16,17,20$; there are obvious periodically reproducing patterns, such as $\overline{1,2}(\bmod 3), \overline{4,2}(\bmod 6)$, or the more recondite, and curiously repetitive,

$$
\overline{6,5,2,2,2,2,4,1,6,6,6,6,5,3,4,4,4,4,1,2,5,5,5,5,3,6,1,1,1,1,2,4,3,3,3,3}
$$

of length 36 corresponding to modulus 7 . We state here a simple consequence of our main continued fraction 42 in Theorem 5 .

Corollary 2. (i) The sequence $\left(\alpha_{n}\right)$ of pseudo-factorials is eventually periodic modulo any integer $M \geq 2$. (ii) For each $m \geq 2$, the sequence $\left(\alpha_{n}\right)$ satisfies modulo $M=3^{\lceil m / 2\rceil} m !^{2}$ a linear recurrence with constant coefficients that is of order at most $m$.

Proof (sketch). The statement is an instance of the general fact that $J$-fraction expansions with integer coefficients automatically imply congruence properties 8 . 
(i) The main continued fraction 42 representing the ordinary generating function $F(z)$ of pseudo-factorials has a factor of $M^{2}$ at its numerator of rank $(M+1)$. In particular, the contributions induced by the stages $(M+1),(M+2)$, and so on, of this continued fraction are zero modulo $M^{2}$. In other words, $F(z)$ is congruent modulo $M^{2}$ to the $M$ th convergent of the continued fraction. Thus, it satisfies, modulo $M^{2}$, a linear recurrence of order at most $M$. Hence it is eventually periodic modulo $M$.

(ii) The estimate above of the order of the recurrence satisfied by modular reductions of the pseudo-factorials can be vastly improved $[8$. By the classical $(2 \times 2)-$ determinant identity of orthogonal polynomials and convergent denominators, the difference of two successive convergents of (32) satisfies the identity

$$
\frac{P_{k+1}(z)}{Q_{k+1}(z)}-\frac{P_{k}(z)}{Q_{k}(z)}=\frac{a_{1} a_{2} \cdots a_{k} z^{2 k}}{Q_{k}(z) Q_{k+1}(z)} .
$$

This specializes to the $J$-fraction (42) relative to $F(z)$, when the $a_{j}$ are taken to be as in 43 . By expressing that $F(z)$ is the sum of the differences of its successive convergents, we then obtain, for any $m \geq 0$,

$$
F(z)=\frac{P_{m}(z)}{Q_{m}(z)}+\sum_{k \geq m} \frac{a_{1} a_{2} \cdots a_{k} z^{2 k}}{Q_{k}(z) Q_{k+1}(z)} .
$$

In particular, since the $a_{j}$ are all integers and the $Q_{j}$ are integral with $Q_{j}(0)=1$, we have,

$$
F(z) \equiv \frac{P_{m}(z)}{Q_{m}(z)} \quad(\bmod M), \quad \text { with } M=a_{1} a_{2} \cdots a_{m},
$$

in the sense that coefficients of both series are equal, after reduction modulo $M$. Thus, modulo $M$, the $\alpha_{n}$ satisfy a linear recurrence whose characteristic polynomial is exactly the denominator polynomial $Q_{m}(z)$ (reduced $\left.\bmod M\right)$.

As an illustration, corresponding to $m=1,2,3$, we find the congruences

$$
\begin{aligned}
& F(z) \equiv \frac{1}{1+z} \quad\left(\bmod 3 \cdot 1 !^{2}\right), \quad F(z) \equiv \frac{1-z}{1+2 z^{2}} \quad\left(\bmod 3 \cdot 2 !^{2}\right), \\
& F(z) \equiv \frac{1+2 z+z^{2}}{1+3 z+6 z^{2}+10 z^{3}} \quad\left(\bmod 3^{2} \cdot 3 !^{2}\right),
\end{aligned}
$$

which already justify the data of Figure 3 for moduli $2,3,4,6,9,12,18$; for instance, from the convergent $P_{1} / Q_{1}$, we find $\alpha_{n} \equiv(-1)^{n}(\bmod 3)$. For $m=7$, the form

$$
\frac{P_{7}(z)}{Q_{7}(z)} \equiv 5+\frac{3+6 z+5 z^{2}+2 z^{3}+2 z^{4}+2 z^{5}}{1+4 z^{6}} \quad(\bmod 7)
$$

explains the observed patterns of $\left(\alpha_{n}\right)$ modulo 7 and the period equal to 36 . By contrast, for $m=11$, we find that

$$
\frac{P_{11}(z)}{Q_{11}(z)} \equiv 1+10 z+9 z^{2}+2 z^{3}+5 z^{4}+4 z^{5}+10 z^{6}+6 z^{7}+5 z^{8}+z^{9}+z^{10} \quad(\bmod 11)
$$

( $Q_{11}$ reduces to 1 modulo 11 ), thereby establishing that the $\alpha_{n}$ with $n \geq 11$ are all divisible by 11 .

As the previous discussion suggests, congruence properties of pseudo-factorials are tightly linked to arithmetic properties of the $Q$ polynomials whose exponential generating function has been determined in Theorem 6. Let again $\Pi_{r}$ denote an unspecified polynomial of degree $r$. Without attempting a general discussion, 
we only remark here the existence of striking regularities, as summarized by the following data. First, for $m$ a prime of the form $6 \mu+1$ :

\begin{tabular}{cccc}
\hline \hline $\bmod 7$ & $\bmod 13$ & $\bmod 19$ & $\bmod 31$ \\
\hline$\frac{P_{7}}{Q_{7}} \equiv \frac{\Pi_{6}}{1+4 z^{6}}$ & $\frac{P_{13}}{Q_{13}} \equiv \frac{\Pi_{12}}{1+11 z^{12}}$ & $\frac{P_{19}}{Q_{19}} \equiv \frac{\Pi_{18}}{1+11 z^{18}}$ & $\frac{P_{31}}{Q_{31}} \equiv \frac{\Pi_{30}}{1+4 z^{30}}$. \\
\hline \hline
\end{tabular}

Finally, for $m$ a prime of the form $6 \mu+5$ :

\begin{tabular}{cccc}
\hline \hline $\bmod 5$ & $\bmod 11$ & $\bmod 17$ & $\bmod 23$ \\
\hline$\frac{P_{5}}{Q_{5}} \equiv \Pi_{4}$ & $\frac{P_{11}}{Q_{11}} \equiv \Pi_{10}$ & $\frac{P_{17}}{Q_{17}} \equiv \Pi_{16}$ & $\frac{P_{23}}{Q_{23}} \equiv \Pi_{22}$. \\
\hline \hline
\end{tabular}

\section{Conclusion}

The relation between elliptic functions and continued fractions is an old subject, one that is especially rich. Connections are manifest with the theta function framework in the form of various types of $q$-series expansions, starting with Eisenstein and including the celebrated Rogers-Ramanujan identities [1, Ch. 16]. Closer to our perspective are early contributions due to Stieltjes and Rogers regarding the Jacobian sn,cn framework. Conrad, first in his dissertation [5] then in collaboration with Flajolet [6], has elicited new connections with the Dixonian framework of the sm,cm functions. As the present work supplemented by further investigations of ours indicate, there are new continued fractions to be explored, attached to the Weierstraß and Dixonian frameworks. In this vein, we have recently discovered new elliptic continued fractions, relative to "equiharmonic" and "lemniscatic" numbers, which are lattice analogues of the pseudo-factorials - we plan to report on these in a future publication.

Acknowledgements. The work of P. Flajolet was supported in part by the SADA and LAREDA Projects of the French National Research Agency (ANR). The authors are grateful to Frédéric Chyzak, Manuel Kauers, and Bruno Salvy for insightful discussions relative to computer verification of identities. They are also indebted to Bruno Salvy for making available his upgraded version of the MAPLE Gfun package on which the developments of Section 8 could be most effectively built. Thanks finally to Robin Chapman, an anonymous referee, and the editor for encouraging remarks and constructive suggestions.

\section{REFERENCES}

[1] Bruce C. Berndt. Ramanujan's Notebooks, Part III. Springer Verlag, 1991.

[2] Leonard Carlitz. Some orthogonal polynomials related to elliptic functions. Duke Mathematical Journal, 27:443-459., 1960.

[3] T. S. Chihara. An Introduction to Orthogonal Polynomials. Gordon and Breach, 1978.

[4] Frédéric Chyzak and Bruno Salvy. Non-commutative elimination in Ore algebras proves multivariate identities. Journal of Symbolic Computation, 26(2):187-227, 1998.

[5] Eric van Fossen Conrad. Some Continued Fraction Expansions of Laplace Transforms of Elliptic Functions. PhD Thesis, The Ohio State University, 2002.

[6] Eric van Fossen Conrad and Philippe Flajolet. The Fermat cubic, elliptic functions, continued fractions, and a combinatorial excursion. Séminaire Lotharingien de Combinatoire, 54(B54g):1-44, 2006.

[7] Alfred Cardew Dixon. On the doubly periodic functions arising out of the curve $x^{3}+y^{3}-$ $3 \alpha x y=1$. The Quarterly Journal of Pure and Applied Mathematics, 24:167-233, 1890. 
[8] Philippe Flajolet. On congruences and continued fractions for some classical combinatorial quantities. Discrete Mathematics, 41:145-153, 1982.

[9] Jacek Gilewicz, Elie Leopold, Andreas Ruffing, and Galliano Valent. Some cubic birth and death processes and their related orthogonal polynomials. Constructive Approximation, 24(1):71-89, 2006.

[10] Adolf Hurwitz. Über die Entwicklungscoefficienten der lemniskatischen Functionen. Nachrichten von der Gesellschaft der Wissenschaften zu Göttingen, MathematischPhysikalische Klasse, pages 273-276, 1897.

[11] Adolf Hurwitz. Über die Entwickelungscoefficienten der lemniskatischen Functionen. Mathematische Annalen, 51:196-226, 1899.

[12] Mourad E. H. Ismail. Classical and Quantum Orthogonal Polynomials in One Variable. Number 98 in Encyclopedia of Mathematics and its Applications. Cambridge University Press, 2005.

[13] Mourad E. H. Ismail and D. Masson. Some continued fractions related to elliptic functions. In B. Berndt and F. Gesztesy, editors, Continued Fractions: from Analytic Number Theory to Constructive Approximation, volume 236 of Contemporary Mathematics, pages 149-166, Providence, RI, 1999. AMS.

[14] Mourad E. H. Ismail, Galliano Valent, and Gang J. Yoon. Some orthogonal polynomials related to elliptic functions. Journal of Approximation Theory, 112(2):251-278, 2001.

[15] Peter Lindqvist and Jaak Peetre. Generalized trigonometric functions (solution to problem 10744). The American Mathematical Monthly, 108(5):473-474, 2001.

[16] Peter Lindqvist and Jaak Peetre. Two remarkable identities, called twos, for inverses to some Abelian integrals. The American Mathematical Monthly, 108(5):403-410, 2001.

[17] J. S. Lomont and John Brillhart. Elliptic polynomials. Chapman \& Hall/CRC, Boca Raton, FL, 2001.

[18] Erik Lundberg. Om hypergoniometriska funktioner af komplexa variabla. Manuscript, 1879. English translation, "On hypergoniometric functions of complex variables" available from Jaak Peetre's web page.

[19] J. Meixner. Orthogonale Polynomsysteme mit einer besonderen Gestalt der erzeugenden Funktion. Journal of the London Mathematical Society, 9:6-13, 1934.

[20] Stephen C. Milne. Infinite families of exact sums of squares formulas, Jacobi elliptic functions, continued fractions, and Schur functions, volume 5 of Developments in Mathematics. Kluwer Academic Publishers, Boston, MA, 2002. Reprinted from Ramanujan J. 6 (2002), no. $1,7-149$.

[21] Oskar Perron. Die Lehre von der Kettenbrüchen, volume 2. Teubner, 1957.

[22] F. Pollaczek. Sur une généralisation des polynomes de Jacobi. Mémorial des Sciences Mathématiques, CXXI:55p, 1956.

[23] Tanguy Rivoal. Approximations rationnelles des valeurs de la fonction gamma aux rationnels. Preprint, 17 pages. Available fom the author's home page, January 2009.

[24] L. J. Rogers. On the representation of certain asymptotic series as convergent continued fractions. Proceedings of the London Mathematical Society (Series 2), 4:72-89, 1907.

[25] Bruno Salvy and Paul Zimmermann. GFUN: a Maple package for the manipulation of generating and holonomic functions in one variable. ACM Transactions on Mathematical Software, 20(2):163-167, 1994.

[26] N. J. A. Sloane. The On-Line Encyclopedia of Integer Sequences. 2008. Published electronically at www.research.att.com/ ${ }^{n j}$ as /sequences/.

[27] Richard P. Stanley. Differentiably finite power series. European Journal of Combinatorics, 1:175-188, 1980.

[28] Richard P. Stanley. Enumerative Combinatorics, volume II. Cambridge University Press, 1999.

[29] T. Stieltjes. Sur la réduction en fraction continue d'une série procédant selon les puissances descendantes d'une variable. Annales de la Faculté des Sciences de Toulouse, 4:1-17, 1889.

[30] Alfred van der Poorten. A proof that Euler missed ... Apéry's proof of the irrationality of $\zeta(3)$. Mathematical Intelligencer, 1:195-203, 1979.

[31] H. S. Wall. Analytic Theory of Continued Fractions. Chelsea Publishing Company, 1948.

[32] E. T. Whittaker and G. N. Watson. A Course of Modern Analysis. Cambridge University Press, fourth edition, 1927. Reprinted 1973. 
[33] Doron Zeilberger. A holonomic systems approach to special functions identities. Journal of Computational and Applied Mathematics, 32:321-368, 1990.

R.B.: Institut Fourier (CNRS UMR 5582), 100 rue des Maths, Université de Grenoble I, F-38402 Saint Martin d'Hères, France.

P.F.: Algorithms, INRIA-Rocquencourt, F-78150 Le Chesnay, France 